\title{
BMJ Open Using the theoretical domains framework and the behavioural change wheel in an overarching synthesis of systematic reviews
}

\author{
Michelle Richardson, ${ }^{1}$ Claire Louise Khouja, ${ }^{2}$ Katy Sutcliffe, ${ }^{1}$ James Thomas $^{1}$
}

To cite: Richardson M, Khouja CL, Sutcliffe K, et al. Using the theoretical domains framework and the behavioural change wheel in an overarching synthesis of systematic reviews. BMJ Open 2019;9:e024950. doi:10.1136/ bmjopen-2018-024950

- Prepublication history and additional material for this paper are available online. To view please visit the journal (http:// dx.doi.org/10.1136/bmjopen2018-024950).

Received 22 June 2018 Revised 25 March 2019 Accepted 21 May 2019

Check for updates

(C) Author(s) (or their employer(s)) 2019. Re-use permitted under CC BY-NC. No commercial re-use. See rights and permissions. Published by BMJ.

${ }^{1}$ University College London Social Science Research Unit, London, UK

${ }^{2}$ University of York, York, UK

Correspondence to Dr Michelle Richardson; m.richardson@ucl.ac.uk

\section{ABSTRACT}

Objective Synthesis that can filter the evidence from multiple sources to inform the choice of intervention components is highly desirable yet, at present, there are few examples of systematic reviews that explicitly define this type of synthesis using behaviour change frameworks Here, we demonstrate how using the Theoretical Domains Framework (TDF) and the Behaviour Change Wheel (BCW) made it possible to bring together the findings from a series of three interconnected systematic reviews on the self-care of minor ailments (MAs) to inform the choice of intervention components.

Method The TDF and the capability, opportunity, motivation model of behaviour at the hub of the BCW were used to synthesise the findings from the three reviews, including syntheses of service-user views in interviews (review 1, 20 studies) and surveys (review 2, 13 studies), and evaluations of a range of interventions and services (review 3, 21 studies).

Results The TDF and BCW approach provided a systematic, structured and replicable methodology for retrospectively integrating different types of evidence within a series of systematic reviews. Several intervention strategies, grounded in theory and discussed with key stakeholders, were suggested, which can be implemented and tested.

Conclusions This novel application of the TDF/BCW approach illustrates how it can be used to bring together quantitative and qualitative evidence to better understand self-care behaviour for MAs within a systematic review context. The TDF/BCW approach facilitated exploration of the contradictions and gaps between the separate review syntheses, and supported the identification of possible intervention strategies, grounded in theory. The ongoing development and refinement of this method is supported. PROSPERO registration number CRD42017071515

\section{INTRODUCTION}

In developing complex (especially behavioural) interventions, the synthesis of multiple types of information, including data from individual trials, surveys and interviews, and systematic reviews of qualitative and quantitative evidence, and other studies, is often helpful, yet there are few established
Strengths and limitations of this study

- A worked example of a systematic, replicable mixed-methods approach to identifying the determinants of the self-care of minor ailments.

- Framing of the determinants, in terms of the Theoretical Domains Framework (TDF) and capability, opportunity, motivation model of behaviour, supported the identification, using the Behaviour Change Wheel (BCW) approach, of potential interventions that target the likely determinants of selfcare behaviour, allowing specific recommendations to be made for intervention design and future research in this area.

- Stakeholder involvement helped to assess the feasibility and acceptability of the results.

- The relative novelty of the TDF/BCW approach, and the inferential and speculative aspects of the analysis, mean that the results should be considered cautiously.

methods of synthesis. By addressing the same question from more than one perspective or technique, the findings from different methods can be compared and contrasted. This process is known as triangulation. ${ }^{1}$ If the findings across the different methods are similar, or reinforce one another, then the findings can be considered more robust than those from each method alone. ${ }^{1}$ Framework synthesis uses an a priori 'framework', and offers a highly structured approach to triangulation. In the context of intervention design, synthesis that can filter the evidence from multiple sources in a way that can inform the choice of intervention components is highly desirable yet, at present, there are few examples of systematic reviews, which explicitly define the methods to inform this type of synthesis using behavioural change frameworks.

Recent developments in the field of behavioural change encourage a systematic approach that has the potential to inform the 
Table 1 Summary of three systematic reviews on seeking help for MAs conducted by Richardson et al

\begin{tabular}{|c|c|c|c|c|c|}
\hline Review $^{3}$ & Focus & Type of data & $\begin{array}{l}\text { Conceptual } \\
\text { synthesis }\end{array}$ & Data synthesis & $\begin{array}{l}\text { Quality of primary } \\
\text { studies }\end{array}$ \\
\hline $\begin{array}{l}\text { Interviews (review } \\
1,20 \text { studies) }\end{array}$ & $\begin{array}{l}\text { Service-users' attitudes } \\
\text { towards and experiences } \\
\text { of seeking help for MAs, as } \\
\text { specified by Pillay et al. } .^{19}\end{array}$ & Qualitative & TDF & $\begin{array}{l}\text { Framework synthesis using } \\
\text { the TDF }{ }^{8} \text {; followed by } \\
\text { Content analysis }{ }^{20} \text { within } \\
\text { each TDF domain. }\end{array}$ & $\begin{array}{l}16 \text { low risk (although } \\
\text { the data were mainly } \\
\text { descriptive); } 4 \\
\text { medium risk. }\end{array}$ \\
\hline $\begin{array}{l}\text { Surveys (review } 2 \text {, } \\
13 \text { studies) }\end{array}$ & & Quantitative & TDF & $\begin{array}{l}\text { Framework synthesis using } \\
\text { the TDF, followed by Content } \\
\text { analysis }{ }^{20} \text { within each TDF } \\
\text { domain. Data were then } \\
\text { pooled, where possible, to } \\
\text { calculate weighted mean } \\
\text { percentages (otherwise data } \\
\text { from single surveys were } \\
\text { reported). }\end{array}$ & $\begin{array}{l}4 \text { medium risk; } 9 \text { high } \\
\text { risk. }\end{array}$ \\
\hline $\begin{array}{l}\text { Evaluations } \\
\text { (review } 3,21^{\text {studies)* }}\end{array}$ & $\begin{array}{l}\text { The effectiveness of } \\
\text { interventions/services to } \\
\text { promote self-care for MAs, } \\
\text { as specified by Pillay et al. }{ }^{19}\end{array}$ & Quantitative & $\begin{array}{l}\text { These evaluations } \\
\text { were categorised into } \\
\text { intervention, service } \\
\text { and outcome types. }\end{array}$ & $\begin{array}{l}\text { Narrative synthesis in terms } \\
\text { of intervention and outcome } \\
\text { (health-service use at GP and } \\
\text { A\&E, or symptom reduction }\end{array}$ & $\begin{array}{l}14 \text { studies high risk; } 7 \\
\text { low risk. }\end{array}$ \\
\hline
\end{tabular}

${ }^{*}$ Only controlled studies and interventions that directly targeted the service-user were included here. There were 26 studies in the original synthesis.

A\&E, accident and emergency; GP, general practitioner; MAs, minor ailments; TDF, Theoretical Domains Framework.

choice of intervention components through the identification of barriers to change and associated theoretical change processes. Here, we report an extended version of an overarching synthesis of three interconnected systematic reviews, undertaken by our team on the self-care of minor ailments (MAs). ${ }^{3}$ These reviews, summarised in table 1 (and reported in full elsewhere) were syntheses of service-user views in interviews (review 1) and surveys (review 2), and evaluations (review 3 ) of a range of interventions and services. The overarching synthesis used the Theoretical Domains Framework (TDF) ${ }^{4}$ and the Behaviour Change Wheel $(\mathrm{BCW})^{5}$ to bring the reviews together. $^{3}$

MAs have been defined as non-serious medical conditions (such as a sore throat or cough) that people can care for by themselves using over-the-counter medicines, with support from friends or family, or from self-care services, such as community pharmacy, walk-in/urgent care centres or telephone triage, such as National Health Service (NHS) 111 (a non-emergency helpline), in the UK. MAs do not require appointments with general practitioners (GPs) or attendance at accident and emergency (A\&E), yet they often place an unnecessary strain on these overstretched services. ${ }^{6}$

\section{Behavioural change}

Behavioural change has been shown to be more effective if interventions are based on principles drawn from evidence and theories of behaviour and behavioural change. ${ }^{7}$ The TDF and BCW are recent developments in this field that provide a systematic and theoretical basis for understanding and changing behaviour. The TDF simplifies 33 theories and 128 constructs, which may explain behavioural change, into 14 domains underpinned by psychological theory. ${ }^{489}$ The 14 validated domains are: (1) knowledge, (2) skills, (3) social/professional role and identity, (4) beliefs about capabilities, (5) optimism, (6) beliefs about consequences, (7) reinforcement, (8) intentions, (9) goals, (10) memory, attention and decision processes, (11) environmental context and resources, (12) social influences, (13) emotion and (14) behavioural regulation. These domains include individual-level factors, such as knowledge and skills (eg, knowledge of self-care services), social factors (eg, social support), and environment and resource factors (eg, cost of treatment). They, therefore, prompt the consideration of a wide range of influences. This is especially pertinent when synthesising primary research within reviews, as retrospective coding requires a sufficiently broad range of domains.

The capability, opportunity, motivation model of behaviour (COM-B) distills the TDF into three key domains that interact to predict behaviour, including people's capability, motivation and opportunities for the behaviour. The COM-B system forms the hub of the $\mathrm{BCW}^{5}$ (shown in figure 1, reproduced from Michie $e t a \tilde{})$ ) and, in conjunction with the next layer of the BCW, can be used to identify potentially relevant intervention functions, based on the salient TDF and COM-B domains. The BCW is, therefore, unique in that it helps to identify which components need to change for the target behaviour(s) to occur, and provides guidance on the strategies that can be used to modify the behaviour.

The TDF has been successfully applied to characterise the determinants of a range of health behaviours (including the management of lower back pain, dementia and smoking cessation), examined through interview and survey studies. ${ }^{10}$ The BCW has also received a lot of interest since its initial 2011 publication, and has been 


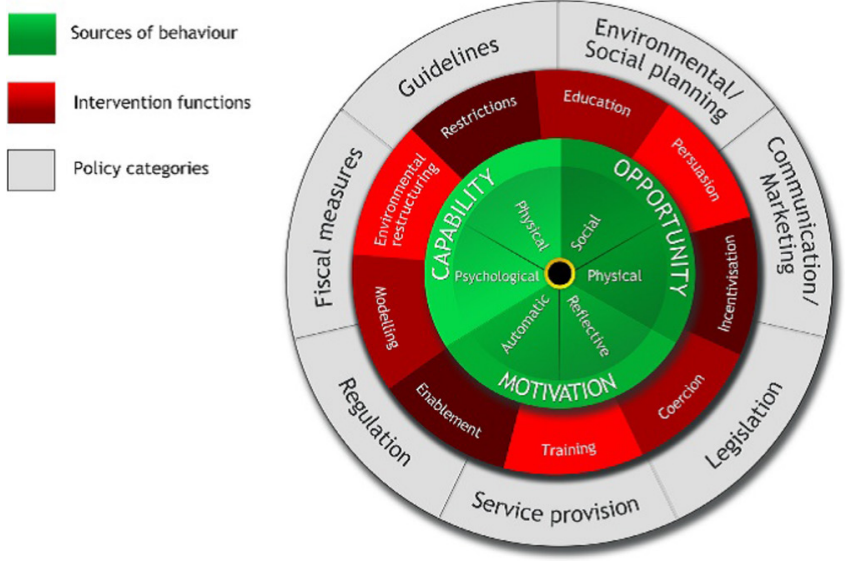

Figure 1 The Behaviour Change Wheel

used to develop or study the implementation of interventions in healthcare settings. ${ }^{11}{ }^{12}$ More recently, these tools are being applied within the context of systematic reviews ${ }^{13}$ to identify the determinants of behaviour and to deconstruct interventions, and link components of interventions with effectiveness. To our knowledge, however, there are no published examples that use the TDF and BCW to bring together qualitative and quantitative evidence within a systematic review. In this proofof-concept study, we used the TDF and BCW to integrate the findings from three systematic reviews that included 54 studies.

\section{AIMS}

This research sought to apply the TDF, the COM-B system of behavioural change and the associated BCW, as tools for bringing together the quantitative and qualitative findings from three systematic reviews on self-care for MAs, to inform the choice of intervention components.

\section{METHOD}

\section{Reviews included in the overarching synthesis}

We conducted three syntheses of service-user views in interviews (review 1, 20 studies) and surveys (review 2, 13 studies) that sought to explore the factors that may influence self-care for MAs and evaluations (review 3, 21 studies) ${ }^{3}$ of the effectiveness of behavioural interventions and services that support self-care for MAs (one study was inlcuded in both the interveiw and survey synthesis). These form the basis of the overarching synthesis reported here. These reviews filled an evidence gap identified by a comprehensive search of the literature in 2015, restricted from 2000 onwards (see Richardson $e t a \hat{l}$ for full details). This identified only one systematic review that had examined self-care for MAs in the UK. In this review by Paudyal et al, ${ }^{14} 31$ studies of UK pharmacy-based MA schemes were synthesised.

\section{Overarching synthesis and behavioural analysis}

The findings from the primary reviews ${ }^{3}$ were integrated into three steps. In step 1, the content of the interventions evaluated in review 3 was coded for behavioural change techniques (BCTs); defined using the BCT Taxonomy V.1. (BCTTV1) ${ }^{9}$ BCTs were then mapped onto the TDF framework (see labels and definitions, table 2). In step 2, the findings from each review were mapped on the COM-B system of the BCW and triangulated using the TDF and COM-B systems. In step 3, the most relevant determinants were mapped onto intervention functions (specified by the BCW) to identify BCTs and intervention strategies.

MR was involved in developing the training materials for the BCTTV $1^{15}$ and has experience in training people to use it, as well as in using the $\mathrm{TDF}^{16}$; CLK successfully completed the online BCT training (available at http:// www.bct-taxonomy.com/) and has experience in using a priori frameworks to code data inductively. MR led all the coding and mapping decisions, which were checked by CLK. Discrepancies were discussed and resolved with a third researcher (KS), where necessary. In cases where there was no previous mapping, or the existing mapping needed to be modified to the particular research context, decisions were made by consensus among the team.

Each step is discussed in more detail, below.

\section{How interventions were mapped (step 1)}

The interview (review 1) and survey (review 2) reviews were previously synthesised using the TDF. ${ }^{3}$ To make the third review comparable, the evaluations of the 11 included interventions were mapped onto the TDF. The 10 evaluations of services were not coded for BCTs as the active ingredients concerned provider type (most commonly nurses), delivery format (telephone or face to face), and setting of treatment (general practice or other) rather than treatment.

BCTs were coded for the self-care of MAs (encompassing a range of behaviours) by two researchers (MR and CLK) and the number of papers that each BCT was present in was recorded. All intervention characteristics (including target behaviour(s), population, context, provider, BCTs and control condition) were extracted from the descriptions in the primary manuscripts. BCTs were subsequently coded onto the TDF, drawing on the results from an expert consensus exercise ${ }^{8}$ that linked 12 of the TDF domains to 59 BCTs from the BCTTV1. In three educational interventions with multiple relevant conditions, the one with the best intervention description was included (to avoid double counting).

\section{Tiangulation of findings using the TDF and COM-B system of the BCW (step 2)}

The TDF domains were mapped onto the COM-B system for the syntheses of service-user views in interviews (review 1) and surveys (review 2), and evaluations (review 3) of interventions, then cross-referenced and tabularised. Capability can be physical or psychological and 
Table 2 The Theoretical Domains Framework (TDF)

TDF domain Description

\begin{tabular}{|c|c|}
\hline Knowledge & An awareness of the existence of something. \\
\hline Skills & An ability or proficiency acquired through practice. \\
\hline Social/professional role and identity & $\begin{array}{l}\text { A coherent set of behaviours and displayed personal qualities of an individual in a } \\
\text { social or work setting. }\end{array}$ \\
\hline Beliefs about capabilities & $\begin{array}{l}\text { Acceptance of the truth, reality or validity about an ability, talent or facility that a } \\
\text { person can put to constructive use. }\end{array}$ \\
\hline Optimism & $\begin{array}{l}\text { The confidence that things will happen for the best, or that desired goals will be } \\
\text { attained. }\end{array}$ \\
\hline Beliefs about consequences & $\begin{array}{l}\text { Acceptance of the truth, reality or validity about outcomes of a behaviour in a given } \\
\text { situation. }\end{array}$ \\
\hline Reinforcement & $\begin{array}{l}\text { Increasing the probability of a response by arranging a dependent relationship or } \\
\text { contingency, between the response and a given stimulus. }\end{array}$ \\
\hline Intentions & A conscious decision to perform a behaviour or a resolve to act in a certain way. \\
\hline Environmental context and resources & $\begin{array}{l}\text { Any circumstance of a person's situation or environment that discourages } \\
\text { or encourages the development of skills and abilities, independence, social } \\
\text { competence and adaptive behaviour. }\end{array}$ \\
\hline Social influences & $\begin{array}{l}\text { Those interpersonal processes that can cause an individual to change their } \\
\text { thoughts, feelings or behaviours. }\end{array}$ \\
\hline Emotion & $\begin{array}{l}\text { A complex reaction pattern, involving experiential, behavioural and physiological } \\
\text { elements, by which the individual attempts to deal with a personally significant } \\
\text { matter or event. }\end{array}$ \\
\hline Behavioural regulation & $\begin{array}{l}\text { Anything aimed at managing or changing objectively observed or measured } \\
\text { actions. }\end{array}$ \\
\hline
\end{tabular}

Table reproduced from Cane et al. ${ }^{16}$

represents an individual's ability to carry out behaviour. Opportunity reflects physical (eg, service opening times) and social (eg, norms about taking care of children's health) features of the environment that influences the behaviour. Motivation can be reflective or automatic and characterises the brain processes that drive behaviour. We drew on the links between the TDF and COM-B, identified by a group of experts in a consensus exercise (see page 92 in the BCW guide). ${ }^{17}$

The extent to which the interventions (evaluated in review 3) matched the implied recommendations of the interviews and surveys was analysed, alongside an analysis of whether or not interventions meeting such recommendations proved to be more effective. Statistical information (direction of effect sizes and associated $p$ values of the interventions) was extracted, where relevant, and reported in online supplementary table 1 (see Richardson et al, for full details including effect sizes and confidence intervals $\left.{ }^{3}\right)$.

In the final stage of this step, themes within the matrix were prioritised into salient domains. Themes and subthemes were identified as important, based on a concordance of findings across the reviews. Concordance was defined as being identified in the interviews and among a quarter or more of survey participants, and/or where there was evidence of effectiveness in the evaluations of interventions (review 3).

\section{BCTs and intervention strategies (step 3)}

The next step was to identify the strategies that are likely to be effective in promoting self-care for MAs. Nine intervention functions were specified in the BCW and mapped on to the COM-B domains: education, persuasion, incentivisation, coercion, training, restriction, environmental restructuring, modelling and enablement (see table 2.3 in the BCW guide ${ }^{17}$ ). Using the guidance from the BCW, those intervention functions that were most likely to address the key determinants of self-care behaviour (identified in the previous step) were selected. BCTs that were linked to the intervention functions were identified using the BCTTV $1^{9}$ and the results from an expert consensus exercise that mapped BCTs onto intervention functions (see table 3.3 in the BCW guide ${ }^{17}$ ). BCTs were selected, therefore, to target the most salient determinants of selfcare for MAs identified in interviews, surveys and evaluations of interventions. This process was supported using 


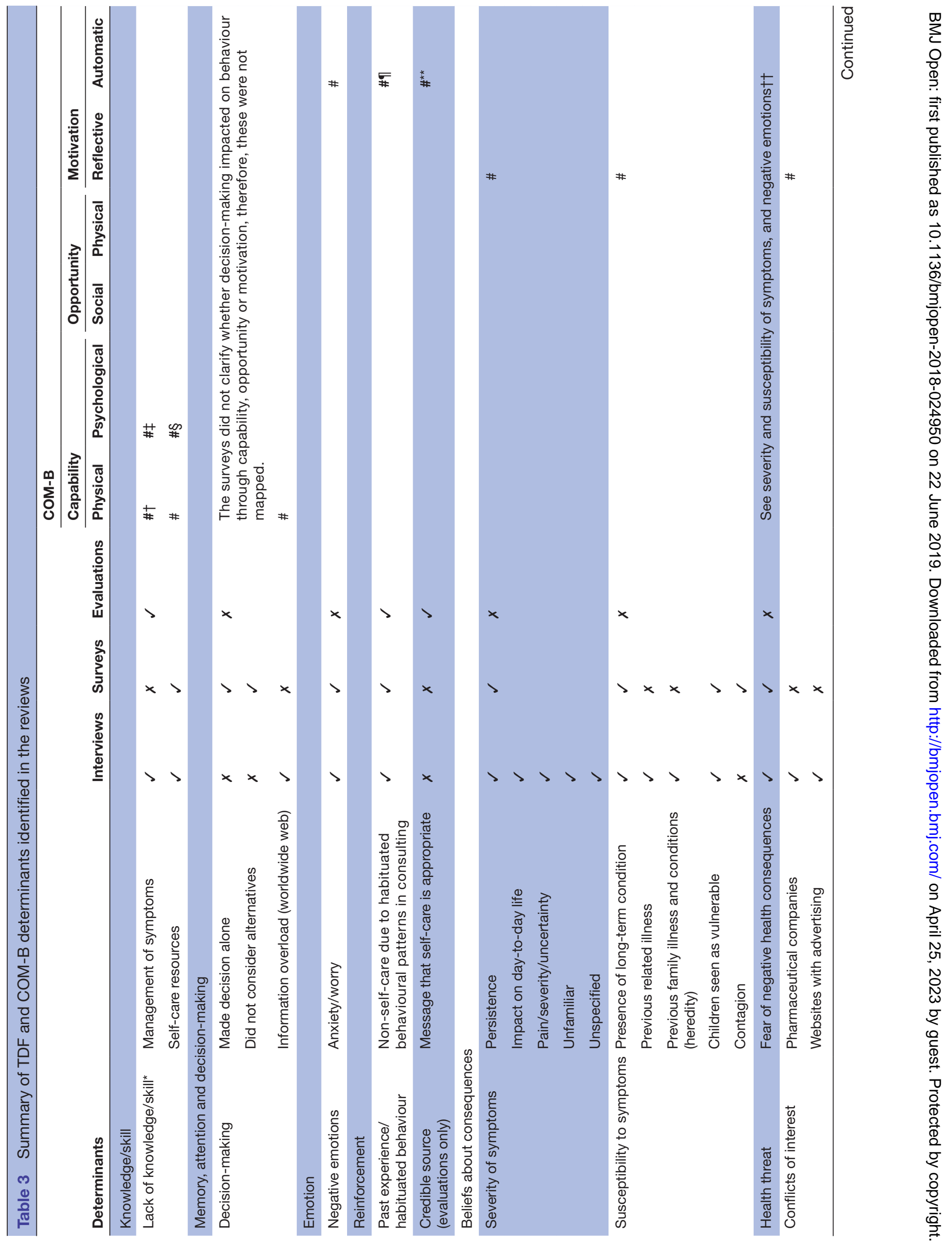




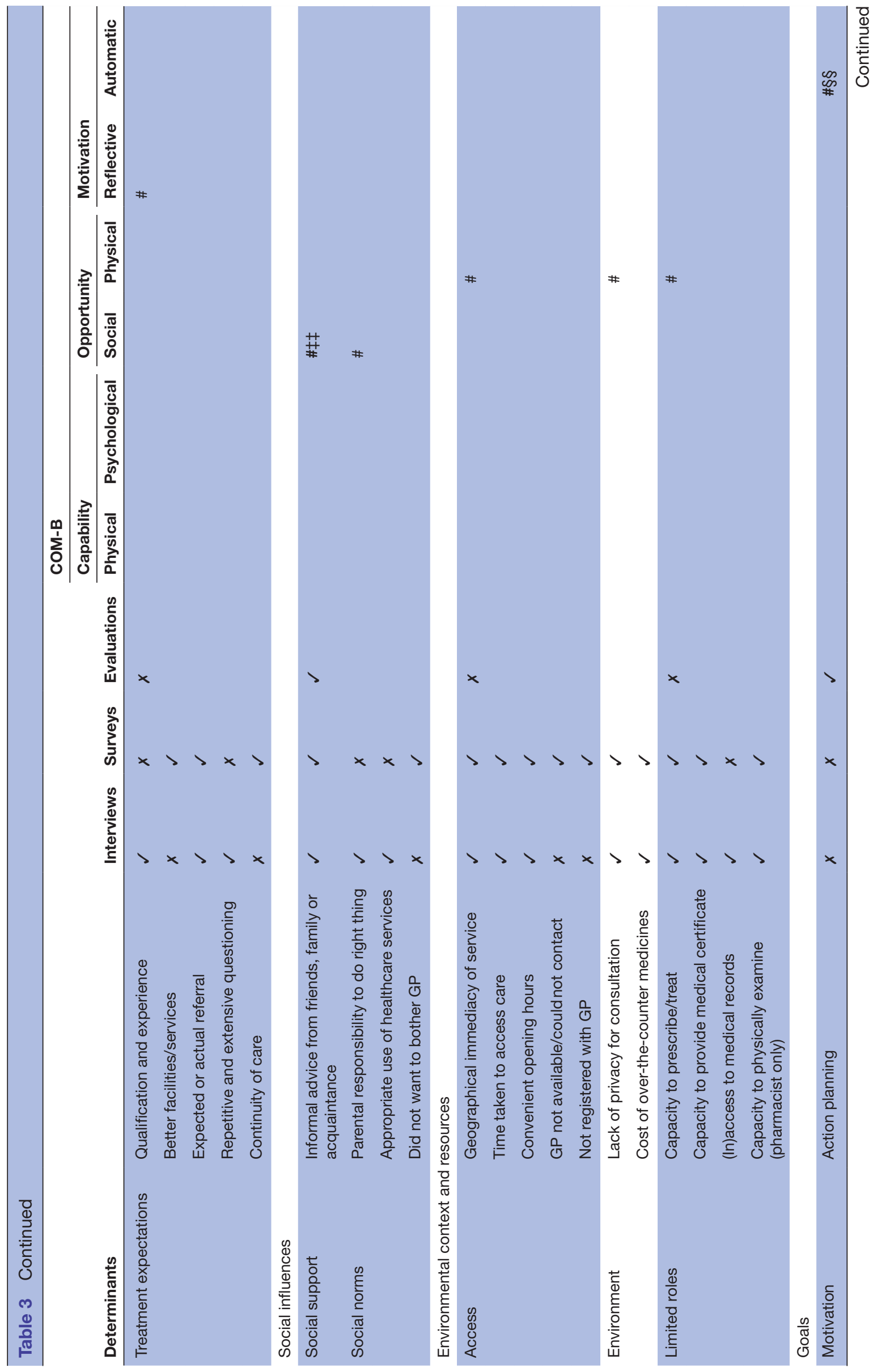

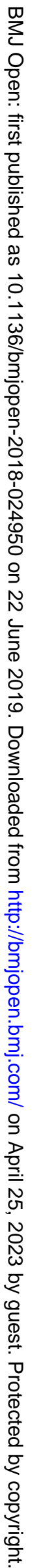




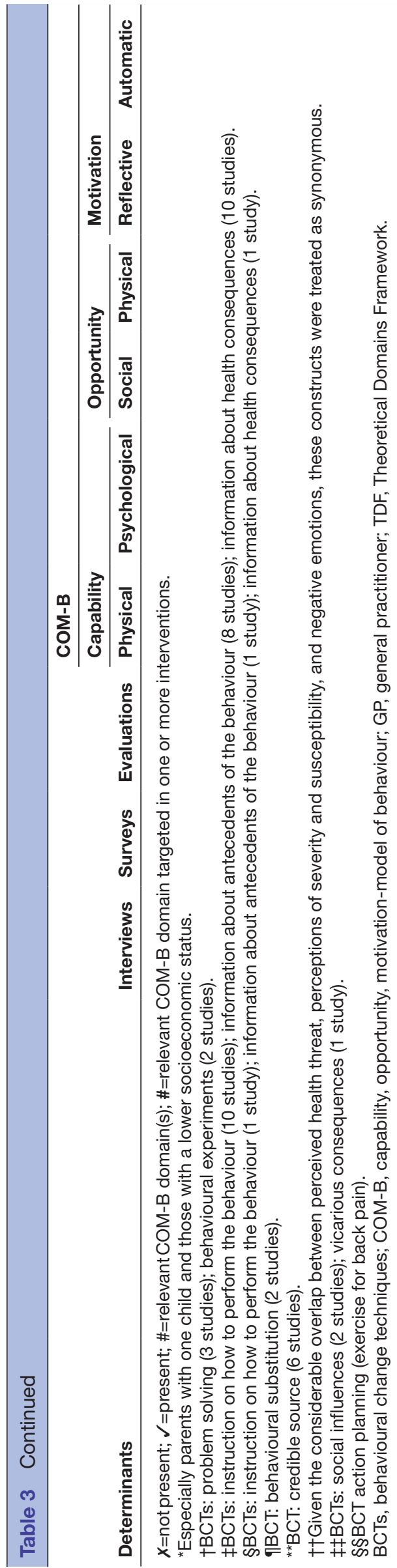

the acceptability, practicability, effectiveness/cost-effectiveness, affordability, safety/side-effects and equity criteria (APEASE) ${ }^{17}$ in consultation with stakeholders (see below), and the results were put into a matrix.

\section{Patient and public involvement}

Stakeholder involvement has been an important aspect of this project, from the early planning stages through to analysis and write-up of the study findings. Stakeholders $(n=5)$ (including representatives from the Department of Health and Social Care (DHSC) policy teams, the DHSC Policy Research Programme, NHS England, and the Economic and Social Research Council) provided feedback on the study protocol and helped to inform the scope of the research topics and research questions. They also met to review the study findings and to prioritise the behavioural change approaches for the self-care of MAs, drawing on the APEASE criteria. While patients and the public were not involved in the design or conduct of the review, patient views were central to the review and its findings as they were the focus of analyses of service-user views (reviews 1 and 2).

\section{RESULTS}

How interventions were mapped: identification of BCTs and TDF domains (step 1)

At the broadest level, interventions were categorised into three groups: health services (10 studies), education (10 studies) and prescribing (3 studies) (Two interventions explored both prescribing and education). Details of intervention characteristics are provided in online supplementary table 1.

\section{Evaluations of services for MAs}

Evaluations of services for MAs were categorised into four types: telephone triage (three studies), walk-in centre (three studies), practice nursing (three studies) and a paramedic service (one study). All of these services included a number of interventions, such as patient examination and diagnosis, provision of advice and treatment, issuing of prescriptions (which usually required a doctor's signature) and referral of the patient to GP/A\&E, where appropriate. With the exception of the community pharmacy interventions, reported elsewhere, ${ }^{14}$ there was little evidence of effectiveness.

\section{Evaluations of interventions for MAs}

Among the educational interventions, five targeted the management of MAs in general, two each for respiratory infections and back pain, and one on conjunctivitis. Nine BCTs were identified; these are detailed in online supplementary table 2, together with illustrative examples from the descriptions of the interventions and the number of interventions that included them. BCTs 'instruction on how to perform the behaviour' and 'information about health consequences' (present in 10 studies each) were most common, followed by 'information 
about antecedents' of self-care behaviour (present in eight studies); then 'credible source' (present in six studies). The remaining BCTs were identified in one to three studies each: 'social support' was identified in the two interventions that focused on respiratory tract infections and 'problem solving' was additionally identified in one of these, 'problem solving' and 'behavioural experiments' were present in the two back pain interventions and 'vicarious learning' was in one study on the management of MAs in general. One of the education interventions for back pain included an exercise component that involved the BCT 'action planning'.

The most frequently occurring BCTs were mapped onto the knowledge TDF domain. Previously, 'instruction on how to perform a behaviour' has been mapped onto the training TDF domain, ${ }^{17}$ however, knowledge was chosen here, as the strategies (such as written guidance on how to treat ailments) emphasised the acquisition of knowledge rather than skills training. The less frequently identified BCTs were mapped onto the following TDF domains: reinforcement ('credible source'), social influences ('social support' and 'vicarious consequences'), training ('problem solving' and 'behavioural experiments') and goals ('action planning'). With the exception of 'problem solving', which was not included in the expert consensus exercise, ${ }^{8}$ these mappings were consistent.

Across educational intervention studies, there was little evidence of beneficial effects across symptoms and consultation outcomes, with almost equal numbers of effect size estimates in a beneficial (10) and in an unfavourable (10) direction and only three (in a beneficial direction) obtaining statistical significance. There were no consistent patterns of intervention effects among those studies that targeted either single or multiple BCTs/TDF domains, suggesting that no single component, or combination thereof, was responsible for effectiveness. Despite the mixed evidence across the reviews, we reasoned that it seems likely that knowledge/skills in symptom management and healthcare services is a necessary, but insufficient, prerequisite to self-care, as has been shown in other health behavioural domains. ${ }^{18}$

Among the prescribing interventions, two BCTs were present (see online supplementary table 2): 'behavioural substitution' (no antibiotic prescribing, identified in three studies) and 'non-specific incentive' (delayed antibiotic prescribing, identified in two studies) which were (both) mapped onto the reinforcement domain, as it was assumed that they worked through weakening the habitual association between minor symptoms and the need to see a GP in the future for similar symptoms, as assessed by subsequent consultation rates. The label of 'non-specific incentive' was relabelled 'incentive' and the definition was modified slightly by replacing the word 'reward' with 'prescription': inform that a prescription will be delivered if and only if there has been effort and/ or progress in performing the behaviour (self-care).

Among the no antibiotic versus immediate prescribing comparisons, three of the five effect size estimates were in a positive direction (one statistically significant); and among the delayed versus control antibiotic comparisons all three effect size estimates were in a positive direction (two obtaining statistical significance).

\section{Triangulation of findings using the TDF and COM-B system of the BCW (step 2)}

Table 3 shows the TDF domains, identified in the reviews (denoted using the symbols ' $\boldsymbol{J}$ ' for present and ' $\boldsymbol{X}$ ' for absent), mapped onto the COM-B model of behaviour (denoted using the '\#' symbol). Where the '\#' is emboldened, this indicates that some of the interventions (identifed in review 3) targeted the focal domain; footnotes are used to denote the BCTs.

Across the interview and survey reviews, 7 of the 14 TDF domains were identified as relevant: Knowledge/Skills (combined in reviews 1 and 2 because they overlapped considerably); memory, attention and decision-making; emotion; reinforcement; beliefs about consequences; social influences and environmental context and resources.

With the exception of two (infrequent) determinants within the memory, attention and decision-making domain (made decision alone and did not consider alternatives), they were successfully mapped onto the COM-B system using the guidance provided. ${ }^{17}$

Three of these seven TDF domains were targeted by existing interventions, including knowledge/skills, reinforcement and social influences. Among the education interventions, while all targeted the combined knowledge/skills domain; only three incorporated elements of skills training ('problem solving' and/or 'behavioural experiments'), the rest targeted only the (psychological) knowledge aspect of the domain. Furthermore, only one intervention targeted knowledge of self-care resources (all focused on management of symptoms). Six education interventions also targeted the reinforcement domain (through the use of a health professional as a credible source to support the intervention); three additionally targeted the social influences domain (social support provided by health professionals and vicarious learning); and one targeted the goals domain using 'action planning' for exercise to help manage back pain.

The prescribing interventions (delayed and none) were mapped onto the reinforcement domain and captured the automatic motivation characteristic of habitual behavioural responses to seek help from a GP or A\&E service when ill.

TDF domains (and subthemes), identified as determinants in the interviews and surveys, were categorised as salient when they were identified in the interviews and among a quarter or more of survey participants, and/or had evidence of effectiveness in the evaluations of interventions. Determinants categorised as salient covered six TDF domains:

- Knowledge/skills (knowledge of services and management of symptoms) (As previously noted, despite the mixed evidence across the evaluations, it seems 


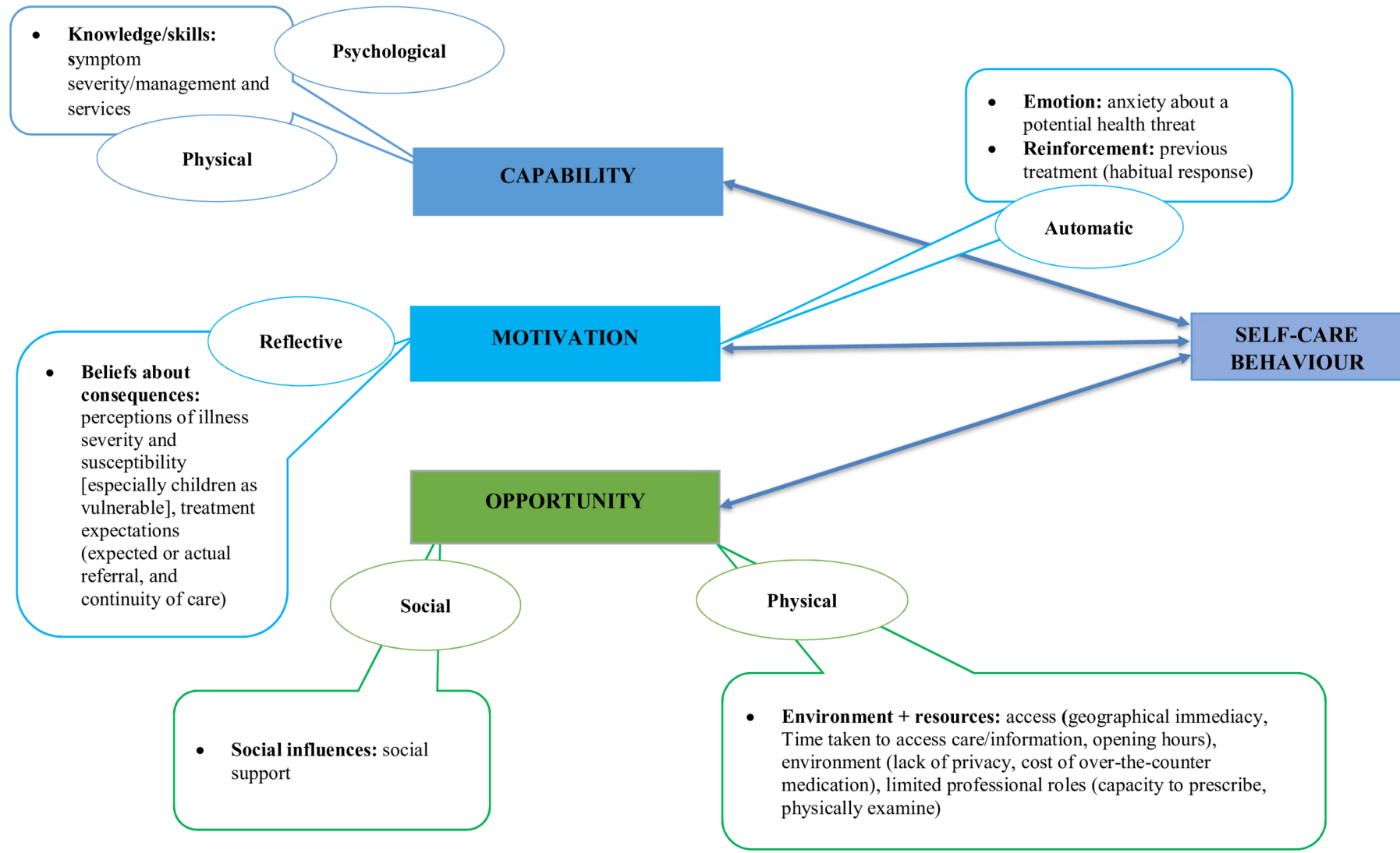

Figure 2 The capability, opportunity, motivation model of behaviour (COM-B) with salient theoretical domains for the self-care of minor ailments

likely that knowledge/skills of services and symptom management is a necessary, but insufficient, prerequisite to self-care).

- Reinforcement (past experience/habituated behaviour).

- Emotion (Anxiety about a potential health threat).

- Beliefs about consequences (perceptions of illness severity, perceptions of illness susceptibility, especially children seen as vulnerable, expected or actual referral and perceived benefits of continuity of care).

- Social influences (social support for self-care).

- Environmental context and resources (geographical immediacy, time taken to access care/information, opening hours, lack of privacy for consultation, cost of over-the-counter medications, limited roles related to prescribing and capacity to physically examine)

Figure 2 shows the salient TDF domains mapped onto the COM-B model of behaviour and indicates that capability (psychological and physical), opportunity (social and environmental) and motivation (reflective and automatic) are all relevant features of self-care behaviour for MAs.

\section{BCTs and intervention strategies (step 3)}

Only three of the six salient TDF domains were targeted by existing interventions, and of these, only antibiotic prescribing strategies (none or delayed targeting the reinforcement domain) showed some evidence of effectiveness, although in two or three studies only. Additional intervention strategies that could be used to target the identified barriers to the self-care of MAs were, therefore, suggested.

Five intervention functions were considered most appropriate for the self-care of MAs in the short term: training, education (in conjunction with training), persuasion, enablement and restriction. One additional function (environmental restructuring) was identified as appropriate for a more long-range approach to intervention. The remaining functions (incentivisation, coercion and modelling) were considered impractical or unacceptable in this context (see online supplementary table 3 ).

Table 4 shows the salient TDF domains, mapped onto the COM-B domains, systematically selected intervention functions, strategies and BCTs to deliver the relevant intervention functions. Illustrative strategies derived from the analyses are briefly discussed for each COM-B domain below.

\section{Capability}

Greater emphasis on knowledge of self-care resources in addition to symptom management may enhance people's capability through improved access to the support they need to self-care effectively. Furthermore, an emphasis on skills training (such as self-monitoring of the behaviour, behavioural rehearsal and demonstration of the behaviour) may improve physical capability 


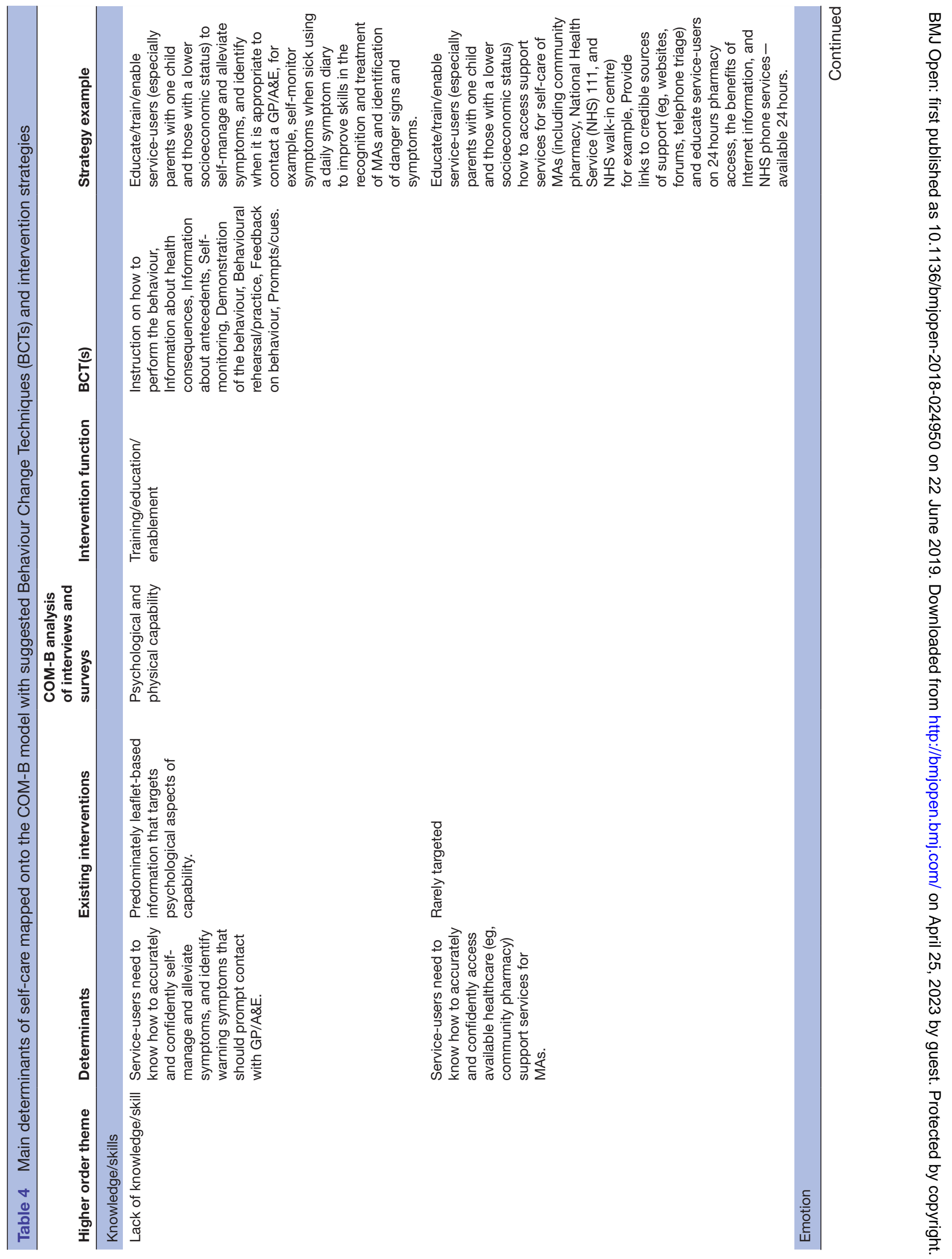




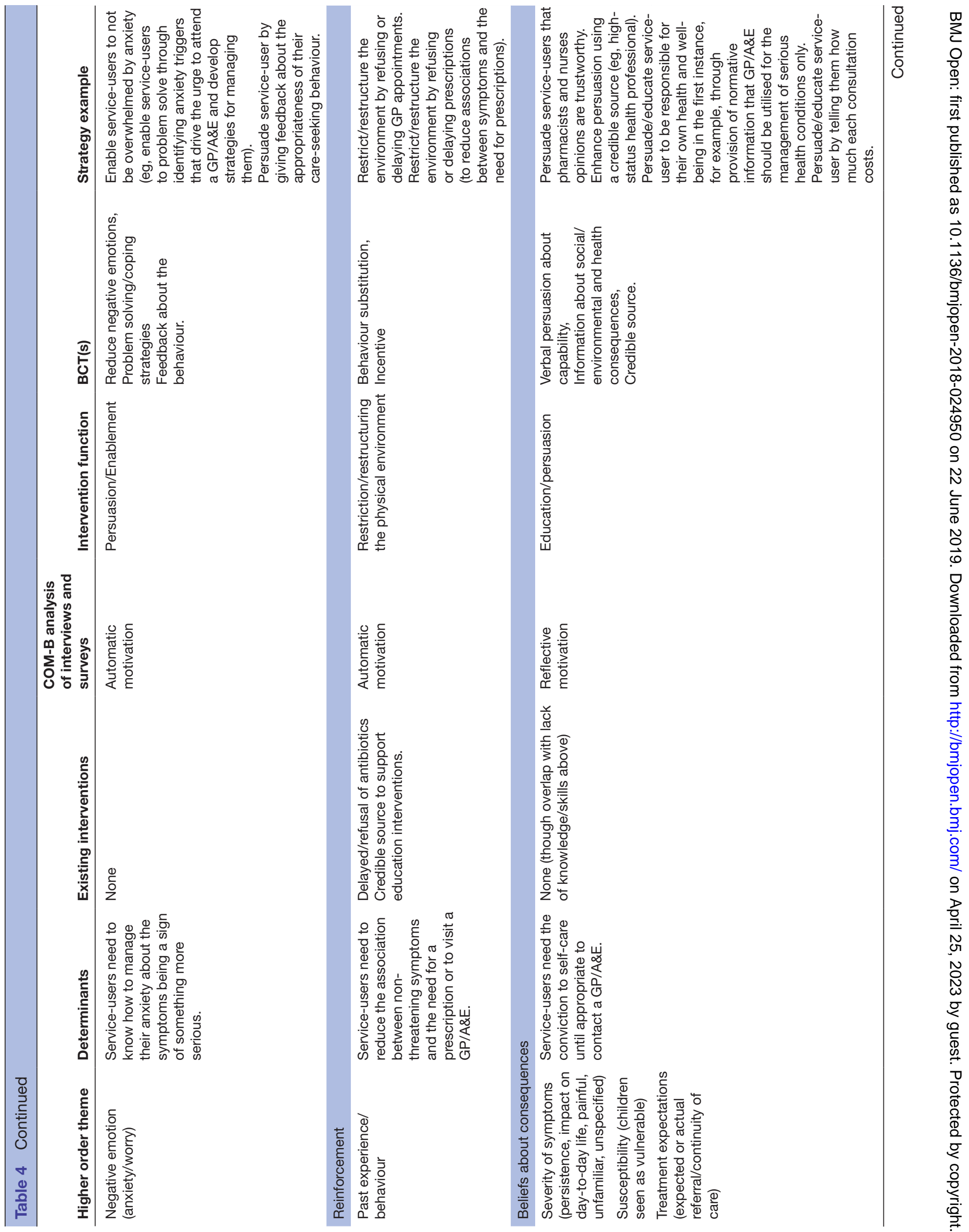




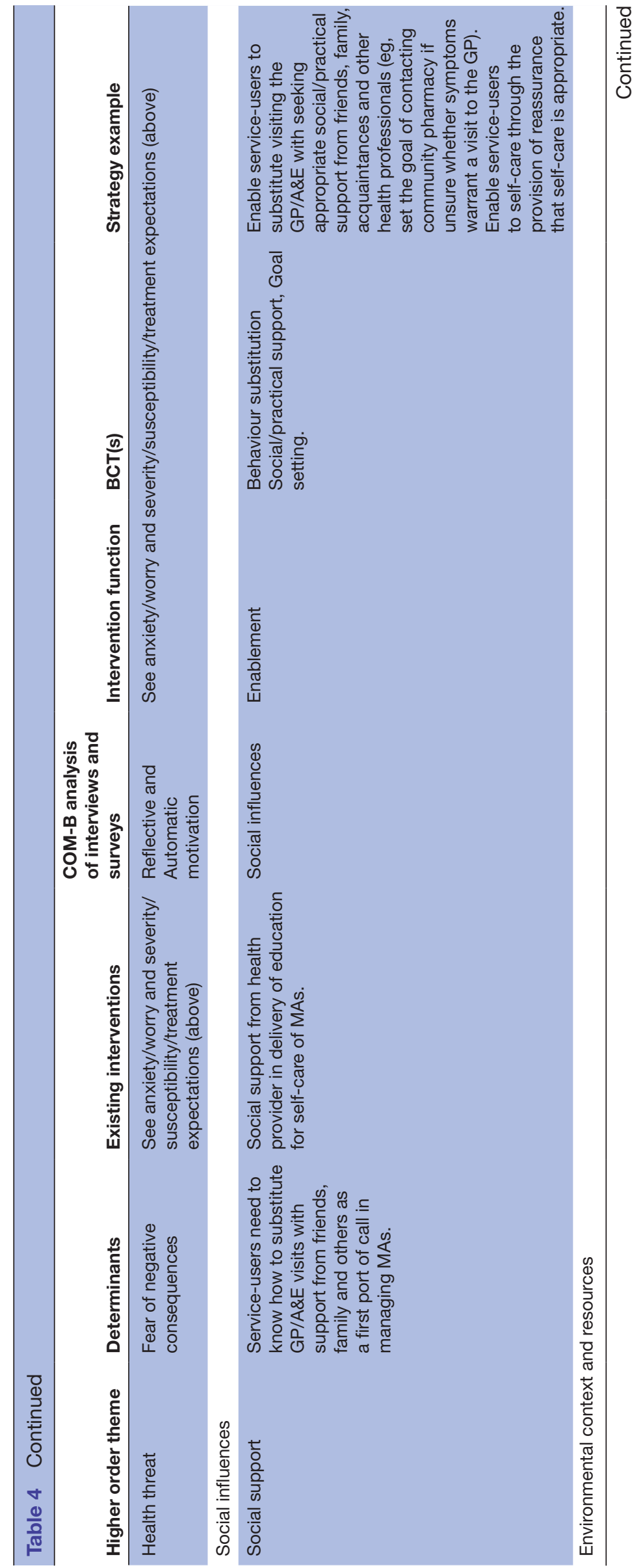

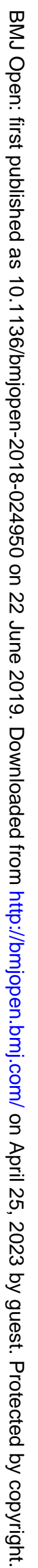




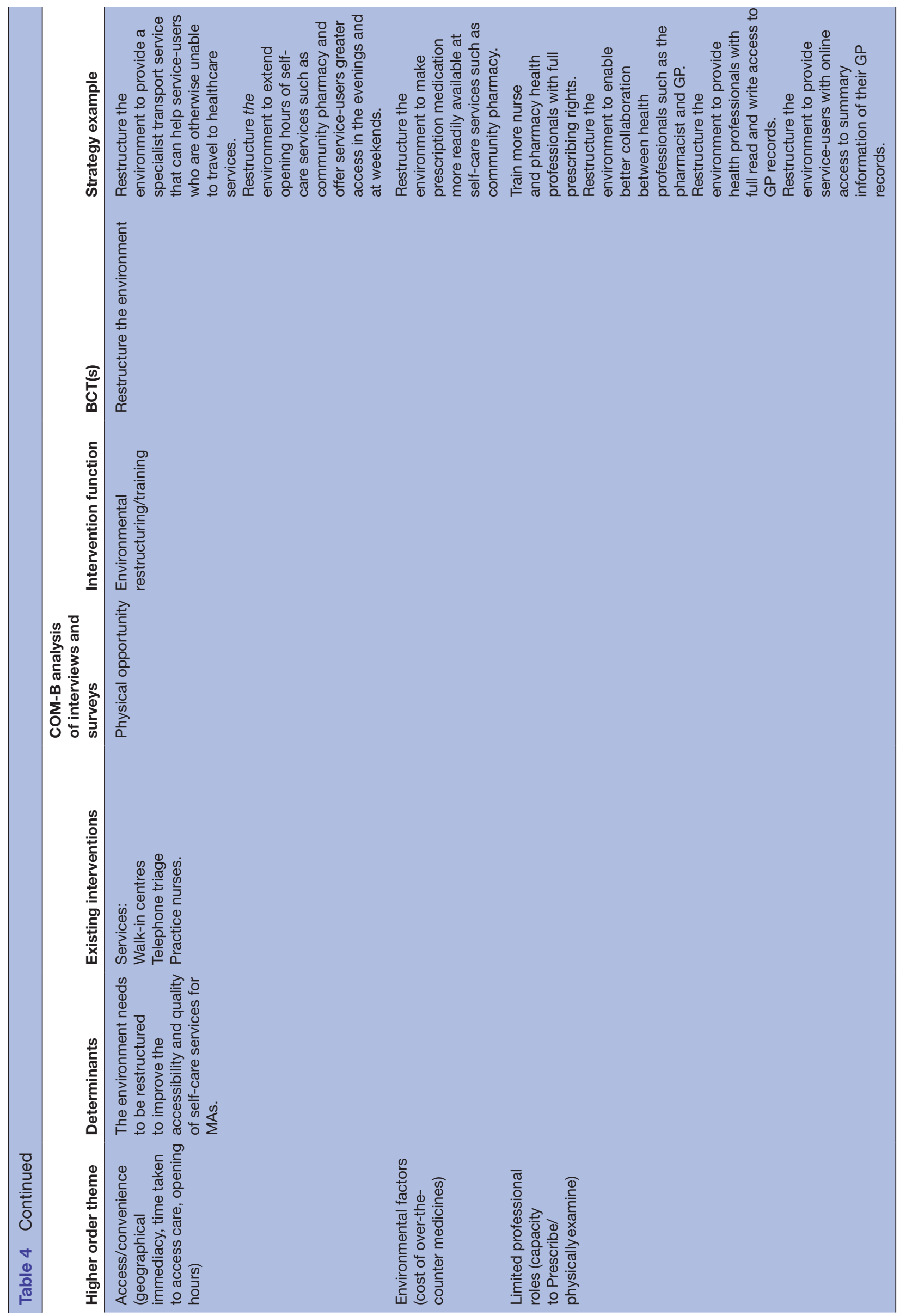


particularly (over and above didactic knowledge acquisition or theory), for example, through using a daily symptom diary, when sick, to improve skills in the recognition and treatment of MAs and the identification of danger signs and symptoms.

\section{Motivation}

In addition to prescribing interventions examined in review 3 (delayed and none), other strategies may be usefully implemented and tested. For example, automatic motivation may be targeted through enabling serviceusers to identify anxiety as a trigger to visit GP/A\&E services and to initiate coping strategies to overcome such urges. In terms of reflective motivation, targeting beliefs about illness severity and susceptibility, especially among parents of children, may be beneficial, for example, using persuasion to strengthen beliefs that the opinions of pharmacists and nurses are trustworthy.

\section{Opportunity}

For social opportunity, a suggested strategy was the enablement of self-care through the provision of reassurance (eg, from a pharmacist) that self-care is appropriate; for physical opportunity, restructuring of the environment was indicated, for example, by training more nurses and pharmacists with full prescribing rights.

\section{Stakeholder involvement}

The stakeholders highlighted that a key NHS priority is to flag the role of pharmacists in managing self-care, and they informed us about strategies that had already been implemented (such as providing private consultation rooms in community pharmacies). Relevant strategies that targeted the community pharmacy were, therefore, emphasised. This is consistent with the findings that pharmacy care for MAs is the only service with some evidence of effectiveness. ${ }^{14}$

\section{DISCUSSION}

\section{Summary of the principal methodological findings}

Framing the determinants of self-care using the TDF and BCW, permitted a synthesis of the findings of three reviews with different types of evidence. The TDF/ BCW approach also helped identify interventions that targeted the likely determinants of self-care behaviour. This enables policy-makers and intervention designers to optimise interventions, by ensuring that they target these likely determinants. Although this method does not guarantee success, it does increase the likelihood of any intervention being successful as it will target the determinants with an intervention that is known to be effective for similar scenarios. Synthesising research in this way effectively creates specific recommendations that can be implemented in various local systems, in ways that build up the evidence of what works, for whom, and under which circumstances. This is an important development distinguishing it from other approaches, such as triangulation, which is limited to the cross-referencing of study findings.

While the BCW provides a systematic and theory-guided method for identifying the types of interventions that are expected to be effective, it does not provide a detailed blueprint for the design of specific behavioural change interventions, as acknowledged by its authors. ${ }^{17}$ For example, we chose to map the BCT (identified in review 3) 'instruction on how to perform the behaviour' onto the TDF Knowledge domain (rather than skills training, as mapped previously) because the content of the interventions emphasised a didactic approach to instruction. Notably, however, the knowledge and skills domains were combined in the interview and survey data, due to considerable overlap. Users, therefore, should expect to apply the framework with some flexibility, as acknowledged by its creators. For example, combining or mapping BCTs onto multiple domains, where there is considerable overlap; adding domains or BCTs, where the model does not account for them; and documenting any such amendments to support the ongoing development and refinement of this method.

As with the TDF, the application of the BCW requires subjectivity and inference. Identifying the intervention functions, and associated BCTs, was fairly straight-forward. However, defining the content of the strategies, while based on the salient determinants in the overarching synthesis, required some creativity. Stakeholder involvement was, therefore, essential for clarifying the feasibility and acceptability of the systematic review and theory-based strategies.

The syntheses required considerable time which was supported by an iterative rather than sequential approach to analyses. In applying this method, the researcher should, therefore, expect to revisit and revise their coding and understanding of the topic several times, as knowledge evolves and emerges throughout the process of synthesis.

\section{Summary of the principal substantive findings}

For people with MAss, mapping the salient TDF domains onto the COM-B system of behavioural change showed that all aspects were relevant for promoting self-care behaviour: people's capability to self-care, their opportunity to self-care and their motivation to self-care. Few of the existing interventions directly targeted these determinants. Potentially relevant intervention strategies that target the salient TDF domains were suggested and discussed with key stakeholders. The existing knowledge-based interventions (evaluated in review 3) did not typically target knowledge of healthcare services (such as community pharmacy) nor skills to support self-care. Given the stakeholders advice to place emphasis on the role of pharmacists in managing self-care, this seems especially important. Health providers, such as pharmacists, may be able to help service-users manage their anxiety (identified as a problem among a high proportion of survey participants). However, service-users 
need to believe that pharmacists' and nurses' opinions are trustworthy. Therefore, strategies to improve trust may be beneficial. Environmental changes, such as improved collaboration between doctors and pharmacists (including full read and write access to GP records), were also identified as potential strategies to implement in the longer term.

\section{Strengths and weaknesses in relation to other reviews}

With the exception of community pharmacy, ${ }^{14}$ no previous systematic reviews were identified in the topic area. To our knowledge, this is the first time that the TDF/BCW approach has been used to bring together evidence using mixed methods within a series of systematic reviews. Limitations include the limited number and diversity of studies in each review; the limited evidence from evaluations (review 3) of interventions targeting the specific determinants identified in the interviews (review 1) and surveys (review 2); and possible biases in the primary reviews, which were conducted by ourselves. The threshold of $25 \%$, used to determine the relevance of determinants in the survey data, was arbitrary. The BCT coding was hampered by the quality of the intervention descriptions, and it was not possible to statistically analyse the individual effectiveness of BCTs or of different combinations. Reducing unnecessary attendance at GP/A\&E services involves multiple behaviours. We were unable, therefore, to map the behavioural change theory to specific behaviour, as has been done elsewhere. Without longitudinal modelling studies or intervention designs, it is difficult to establish which determinants are most important and whether the links between theoretical assessment and BCTs are valid. Nonetheless, consistency across multiple sources provides more confidence in the findings than each method alone. Criteria to assess the quality of outputs when using this type of synthesis would be beneficial, but to our knowledge, are not currently available. Both of the interview and survey reviews were already synthesised using the TDF, whether this approach to synthesis would work if other models had been used is unclear.

\section{Future research}

Given the inferential and speculative aspects of the analysis, and the infancy of the BCW approach, further empirical work is required to check whether the links between theoretical assessments and BCTs are valid. Furthermore, while exploring the inter-relationships between the TDF domains was outside the scope of the review, greater consideration of the inter-relationships between theoretical domains may be warranted. For example, the recursive relationship between environmental and resource factors, and individual perceptions and behaviour, in the decision to self-care.

\section{CONCLUSIONS}

Although the TDF and BCW are being applied more frequently in public health research, to our knowledge, this is the first time that the TDF has been used to bring together quantitative and qualitative evidence in an overarching synthesis, within a series of systematic reviews. The use of the TDF and BCW provided a clear structure, and permitted comparison across reviews and the application of pre-existing mechanistic knowledge within a systematic review context. It also permitted the identification of the salient determinants of the self-care for MAs and potential intervention strategies that target these. The theoretical scaffold provides a means to accumulate the evidence and could potentially be used to understand behaviour in similar contexts.

Acknowledgements We are very grateful to three reviewers (Gillian S Gould, Sarah Denford and Colin Greaves for extremely helpful feedback on our manuscript).

Contributors MR, JT and KS contributed to the conception of this study. MR and CLK were involved in data extraction and synthesis. All authors (MR, CLK, JT and KS) were involved in the writing and the review of the manuscript.

Funding This is an independent report commissioned and funded by the Policy Research Programme in the Department of Health and Social Care.

Disclaimer The opinions expressed in this publication are not necessarily those of the EPPI-Centre, the Centre for Reviews and Dissemination or the funders. Responsibility for the views expressed remains solely with the authors. The views expressed are not necessarily those of the department.

Competing interests None declared.

Patient consent for publication Not required.

Provenance and peer review Not commissioned; externally peer reviewed.

Data sharing statement This overarching synthesis presents previously published data. Please refer to the original report and their authors for these research data.

Open access This is an open access article distributed in accordance with the Creative Commons Attribution Non Commercial (CC BY-NC 4.0) license, which permits others to distribute, remix, adapt, build upon this work non-commercially, and license their derivative works on different terms, provided the original work is properly cited, appropriate credit is given, any changes made indicated, and the use is non-commercial. See: http://creativecommons.org/licenses/by-nc/4.0/.

\section{REFERENCES}

1. O'Cathain A, Murphy E, Nicholl J. Three techniques for integrating data in mixed methods studies. BMJ 2010;341:c4587.

2. Oliver SR, Rees RW, Clarke-Jones L, et al. A multidimensional conceptual framework for analysing public involvement in health services research. Health Expect 2008;11:72-84.

3 Richardson M, Khouja C, Sutcliffe K, et al. Self-care for minor ailments: systematic reviews of qualitative and quantitative research. London: EPPI-Centre, Social Science Research Unit, UCL Institute of Education, University College London, 2018. ISBN: 978-1-91160504-1.

4 Michie S, Johnston M, Abraham C, et al. Making psychological theory useful for implementing evidence based practice: a consensus approach. Qual Saf Health Care 2005;14:26-33.

5 Michie S, van Stralen MM, West R. The behaviour change wheel: a new method for characterising and designing behaviour change interventions. Implement Sci 2011;6:42.

6. Fielding S, Porteous T, Ferguson J, et al. Estimating the burden of minor ailment consultations in general practices and emergency departments through retrospective review of routine data in North East Scotland. Fam Pract 2015;32:165-72.

7. Ammerman AS, Lindquist $\mathrm{CH}$, Lohr KN, et al. The efficacy of behavioral interventions to modify dietary fat and fruit and vegetable intake: a review of the evidence. Prev Med 2002;35:25-41.

8. Cane J, O'Connor D, Michie S. Validation of the theoretical domains framework for use in behaviour change and implementation research. Implement Sci 2012;7:37.

9. Michie S, Richardson M, Johnston M, et al. The behavior change technique taxonomy (v1) of 93 hierarchically clustered techniques: 
building an international consensus for the reporting of behavior change interventions. Ann Behav Med 2013;46:81-95.

10. Beenstock J, Sniehotta FF, White M, et al. What helps and hinders midwives in engaging with pregnant women about stopping smoking? A cross-sectional survey of perceived implementation difficulties among midwives in the North East of England. Implement Sci 2012;7:36.

11. Alexander KE, Brijnath B, Mazza D. Barriers and enablers to delivery of the Healthy Kids Check: an analysis informed by the Theoretical Domains Framework and COM-B model. Implement Sci 2014;9:60.

12. Barker F, Atkins L, de Lusignan S. Applying the COM-B behaviour model and behaviour change wheel to develop an intervention to improve hearing-aid use in adult auditory rehabilitation. Int J Audiol 2016;55(sup3):S90-S98.

13. Heslehurst N, Newham J, Maniatopoulos G, et al. Implementation of pregnancy weight management and obesity guidelines: a metasynthesis of healthcare professionals' barriers and facilitators using the Theoretical Domains Framework. Obes Rev 2014;15:462-86.
14. Paudyal V, Watson MC, Sach T, et al. Are pharmacy-based minor ailment schemes a substitute for other service providers? A systematic review. Br J Gen Pract 2013;63:e472-81.

15. Wood CE, Richardson M, Johnston M, et al. Applying the behaviour change technique (BCT) taxonomy v1: a study of coder training. Transl Behav Med 2015;5:134-48.

16. Cane J, Richardson M, Johnston M, et al. From lists of behaviour change techniques (BCTs) to structured hierarchies: comparison of two methods of developing a hierarchy of BCTs. Br J Health Psychol 2015;20:130-50

17. Michie S, Atkins L, West R. The behaviour change wheel: a guide to designing interventions. Needed: physician leaders 2014;26:146.

18. Abraham C, Conner M, Jones F, et al. Health psychology: Routledge, 2016.

19. Pillay N, Tisman A, Kent T, et al. The economic burden of minor ailments on the National Health Service in the UK. SelfCare 2010;1:105-16.

20. Elo S, Kyngäs $\mathrm{H}$. The qualitative content analysis process. J Adv Nurs 2008;62:107-15. 